\title{
A Self-repairable MEMS Comb Accelerometer
}

\author{
Xingguo Xiong, Yu-Liang Wu*, and Wen-Ben Jone** \\ Department of Electrical and Computer Engineering, \\ University of Bridgeport, Bridgeport, CT 06604, USA \\ Department of Computer Science and Engineering*, \\ The Chinese University of Hong Kong, Shattin, Hong Kong \\ Department of ECECS, University of Cincinnati, Cincinnati, OH 45221, USA** \\ Email:xxiong@bridgeport.edu,ylw@cse.cuhk.edu.hk*,wjone@ececs.uc.edu**
}

\begin{abstract}
In this paper, a built-in self-repair technique for the MEMS comb accelerometer device is proposed. The main device of the comb accelerometer consists of $n$ identical modules, and $m$ modules are introduced as the redundancy. If any of the working module in the main device is found faulty during a built-in self-test (BIST), the control circuit will replace it with a good redundant module. In this way, the faulty device can be self-repaired through redundancy. The implementation of dualmode BIST on the BISR module is discussed. The sensitivity loss due to device modularization can be well compensated by different design alternatives. The yield model for MEMS redundancy repair is developed. The simulation results show that the BISR (built-in self-repair) design leads to effective yield increase compared to non-BISR design, especially for a moderate non-BISR yield. The yield as well as the reliability of the accelerometer can be improved due to the redundancy repair.

Keywords: Microelectromechanical System (MEMS), Built-in self-test (BIST), Built-in self-repair (BISR), comb accelerometer, yield analysis.
\end{abstract}

\section{INTRODUCTION}

As a newly developed discipline, Micro Electro Mechanical System (MEMS) has achieved exciting progress during last decades [1]. All kinds of MEMS devices based upon various working principles have been conceived. MEMS has found broad applications in many areas, and the world MEMS market is growing steadily in a very fast pace. As more and more MEMS devices are used, fault-tolerant MEMS design is extremely crucial with the following three reasons. First, with increasing applications of MEMS to safety-critical fields, such as aerospace, automobile and medical applications, MEMS reliability is becoming a very important issue. Especially, many MEMS devices have movable parts and their repeated movements (vibrations, etc.) may lead to different kinds of structural material fatigues. For example, the friction between the contacting surfaces of movable and fixed parts may wear out the device structure. Thus, even if a MEMS device is tested as fault-free, it still may fail after serving for a certain lifetime. Such a failure during in-field usage is a potential threat especially for safety-critical applications. Second, due to the involvement of multiple fields in MEMS design and fabrication, in contrast to the well-developed VLSI technology, MEMS fabrication is vulnerable to more defect sources. Currently, MEMS device fabrication yield is much lower than that of VLSI circuits. Third, there is increased tendency that MEMS is going to be integrated into system-on-chip (SoC) designs using a standard CMOS process [2]. That is, MEMS devices will be fabricated on the same chip with digital, analog, memory, and FPGA circuit technologies. Fault-tolerant design for traditional CMOS circuits have been proposed and many available techniques are existent [3][4]. However, fault-tolerant design for MEMS devices has never been studied. It will be uneconomical to get rid of the entire SoC chip, if there exist minor MEMS defects. Thus, it is emergent to find a solution to have a defective MEMS device fix itself, whenever the test process (in-field or manufacturing) finds defects existent. By implanting the built-in self-repair (BISR) feature into MEMS devices, the reliability as well as yield rate can be greatly improved.

Efforts on trimming the device geometry parameters with certain physical or chemical processes have been reported. In [5], the highly focalized laser beam is used to precisely trim the geometry parameter of the thin film resistor, thus the resistance can be accurately adjusted to meet the design expectation. If the dimension of the thin film resistor is larger than the designed value, the laser trimming can repair the deviated resistance back to the good value. In [6], the ion milling and isotropic RIE etching techniques are used to reduce the beam width and the beam height of a microgyroscope. Hence, the resonant frequencies in the driving and detection modes can be precisely matched to ensure the proper function of the gyroscope. These can be treated as somewhat the "hard repair" of MEMS devices. However, the methods of [5] [6] have the following disadvantages. First, these efforts are not "self-repair" and they require an extremely precise control over the process to avoid any over-trimming or over-milling. Second, the repairing process may have to be performed for each individual device separately with different adjustment, because each defective device may have its own geometry deviation. This leads to extremely high cost for the device repairing process through trimming, milling or etching, so it is not suitable for batch fabrication processes. Furthermore, the defects that can be repaired are very limited, because only defects involving deviation in geometry parameters can be dealt with. For other defects such as stiction and broken beams, they cannot be applied. 
In this paper, a self-repairable MEMS comb accelerometer based on modular redundancy is proposed. Due to the modularized design, a faulty module can be virtually separated from the main device and replaced with another redundant module, though the defective module is still physically connected to the device. Thus, a defective MEMS device can repair itself given the number of faulty modules is smaller than that of redundant ones. The entire BISR MEMS device is partitioned into $m+n$ identical modules. Among them, $n$ modules serve as the main device, while the other $m$ modules serve as redundant modules. As the prerequisite of BISR, the dual-mode built-in self-test (BIST) of each BISR module is also introduced. The sensitivity loss due to modularized design can be compensated by revising the design parameters such as shrinking the beam width, enlarge the mass width, etc. If the design parameters are fixed, the sensitivity can still be effectively compensated by electrostatic force. The yield model for MEMS redundancy repair is developed. Simulation results demonstrate effective yield increase due to the redundancy repair of the MEMS comb accelerometer. An optimized design of the MEMS BISR accelerometer is suggested and a comparison with the nonBISR device is proposed.

\section{NON-BISR MEMS COMB ACCELEROMETER}

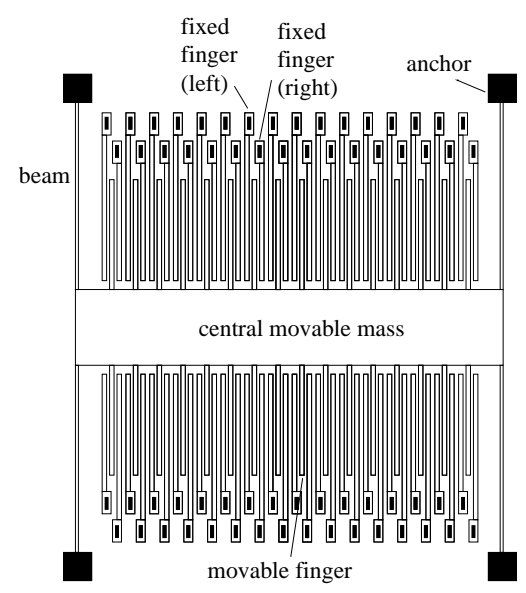

Fig. 1. The general design of MEMS comb accelerometer

A typical surface-micromachined comb accelerometer [7] is shown in Figure 1. The comb accelerometer is made of a thin layer of poly-silicon on the top of a silicon substrate. The thickness of the poly-Si structure layer is about $2 \mu \mathrm{m}$. The fixed portion of the device includes four anchors and many left and right fixed fingers. The movable portion of the MEMS device includes four tether beams, central movable mass and all the movable fingers extruding out of the mass. The entire movable portion is floating about $1.5 \mu \mathrm{m}$ above the substrate. As shown in Figure 1, the central movable mass is connected to the four anchors through four flexible beams. The movable fingers extrude from both sides of the central mass, and can move together with it. There is a pair of fixed fingers around the left and right sides of each movable finger. Each movable finger and its left and right fixed fingers constitute a differential capacitance pair $c_{1}$ and $c_{2}$ separately as shown in Fig. 2. In the static state, each movable finger stays in the middle position between the left and right fixed fingers, and the capacitance gaps of both $c_{1}$ and $c_{2}$ are equal to $d_{0}$. Assume there are $n_{f}$ pairs of finger groups in the MEMS device, and let $C_{1}\left(C_{2}\right)$ represent the sum of all $c_{1}\left(c_{2}\right)$ capacitances. We have

$$
C_{1}=C_{2}=\frac{n_{f} \varepsilon_{0}\left(L_{f}-\Delta\right) h}{d_{0}} .
$$

where $n_{f}$ is the total number of differential capacitance groups, $\varepsilon_{0}$ is the dielectric constant of air, $L_{f}$ is the length of each movable finger, $\Delta$ is the non-overlapped length at the root of each movable finger, and $h$ is the thickness of the device.

Assume the mass of both the central movable mass and all the movable fingers as $M$. If there is an acceleration $a$ in perpendicular to the beams while in parallel to the device plane, the central mass will experience an inertial force $-M \cdot a$. This will result in a certain amount of beam deflection along the direction of the inertial force, hence the equivalent amount of displacement of the central mass and the movable fingers. Thus, each capacitance gap will be changed accordingly which leads to the change of corresponding capacitances (Fig. 2).

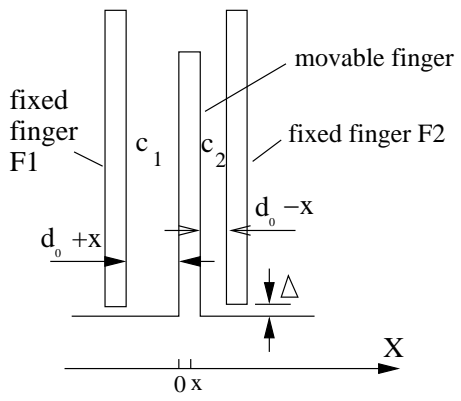

Fig. 2. The schematic diagram of differential capacitance.

As shown in Figure 2, the inertial force results in a deflection of the beams and a certain displacement $x$ of movable fingers along the $X$ direction. Given $x \ll d_{0}$, we have $C_{1}$ and $C_{2}$ changed to [1]

$$
\begin{gathered}
C_{1}=\frac{n_{f} \varepsilon_{0}\left(L_{f}-\Delta\right) h}{\left(d_{0}+x\right)} \approx \frac{n_{f} \varepsilon_{0}\left(L_{f}-\Delta\right) h}{d_{0}}\left(1-\frac{x}{d_{0}}\right), \\
C_{2}=\frac{n_{f} \varepsilon_{0}\left(L_{f}-\Delta\right) h}{\left(d_{0}-x\right)} \approx \frac{n_{f} \varepsilon_{0}\left(L_{f}-\Delta\right) h}{d_{0}}\left(1+\frac{x}{d_{0}}\right) .
\end{gathered}
$$

In order to sense the displacement $x$ of the movable plate M due to the inertial force, modulation voltages $V_{m p}$ and $V_{m n}$ are applied to left and right fixed fingers (F1 and F2) separately

$$
\begin{gathered}
V_{F 1}=V_{m p}=V_{0} \operatorname{sqr}(\omega t), \\
V_{F 2}=V_{m n}=-V_{0} \operatorname{sqr}(\omega t) .
\end{gathered}
$$

where $V_{0}$ represents the modulation voltage amplitude, $\omega$ denotes the frequency of the modulation voltage, and $t$ gives the time for operation. According to the charge conservation law, the charge in capacitances $C_{1}$ and $C_{2}$ must be equal, so 
we have

$$
C_{1}\left(V_{F 1}-V_{M}\right)=C_{2}\left(V_{M}-V_{F 2}\right) .
$$

where $V_{M}$ is the voltage level sensed by the movable plate M. Solving the above equations, we have

$$
V_{M}=\left(x / d_{0}\right) V_{0} \operatorname{sqr}(\omega t) .
$$

It can be observed from this result that under the above modulation voltage biasing, the central movable plate $\mathrm{M}$ acts just as a voltage divider between the top and bottom fixed plates $\mathrm{F} 1$ and $\mathrm{F} 2$ respectively. By measuring the voltage level on central movable electrode $V_{M}$, we can find the displacement $x$ of the central movable plate $\mathrm{M}$, which in turn is directly proportional to the experienced acceleration. Thus, we can derive the value of the applied acceleration along the sensitive direction (X direction). This is the working principle for most differential capacitive MEMS devices.

\section{BISR MEMS COMB ACCELEROMETER DESIGN}

Due to the tiny size (in the range of microns) of MEMS accelerometers, its overall capacitance is generally below $1 \mathrm{pF}$, and the capacitance change in working mode is in range of fF. In order for the tiny capacitance to be detected by the signal sensing circuit, it is desirable to enlarge the device capacitance. Thus, a MEMS comb accelerometer device generally contains large number of repeated comb finger groups in a very compact manner. For example, an ADXL50 accelerometer contains 42 differential comb finger groups [7], while an ADXL150 accelerometer contains 54 differential comb finger groups [8]. A bulk-micromachined comb accelerometer [9] is shown in Figure 3. It contains 80 differential comb fingers groups. Such a highly dense comb structure with many long and narrow capacitance gaps is extremely vulnerable to various defects such as particle contamination, stiction [10]. Taking the ADXL50 accelerometer as an example, the length of each movable finger is $120 \mu \mathrm{m}$, while the capacitance gap between each pair of fixed and movable fingers is only $1.3 \mu \mathrm{m}$. If a conductive particle with diameter larger than $1.3 \mu \mathrm{m}$ falls into any of the 84 capacitance gaps, it will lead to a short-circuit of the device capacitance and result in a failure of the entire device. Thus, a large number of finger groups unavoidably leads to the decrease in yield as well as reliability.

Yield and reliability have been two major concerns for MEMS commercialization. In order to enhance the yield and reliability of MEMS comb accelerometer, a self-repairable MEMS comb accelerometer design is proposed, as shown in Figure 4. Here, the device consists of six identical modules, and each module has its own beams, mass and finger structures (fixed and movable). By assumption, four modules are connected together as the main device, while the remaining two modules serve as redundancy. The movable parts of each module are physically connected to those of adjacent modules through the common anchors, and signals sensed by all movable fingers in the device are connected to the sensing circuit directly. However, the fixed fingers of each

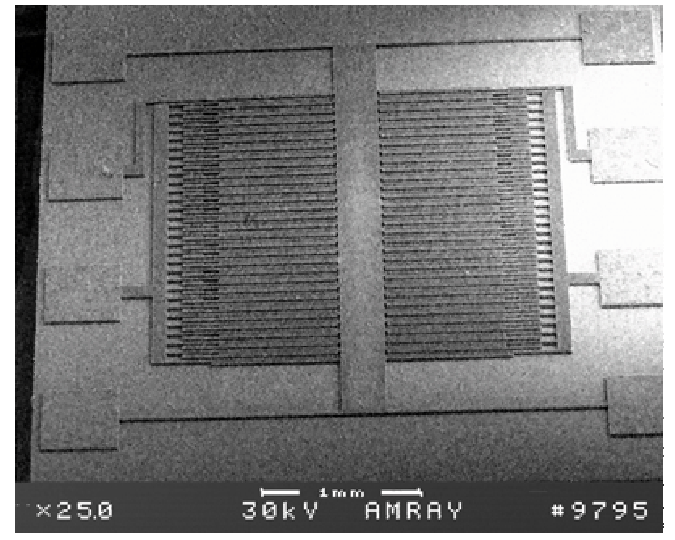

Fig. 3. SEM photo of a bulk-micromachined comb accelerometer device[9].

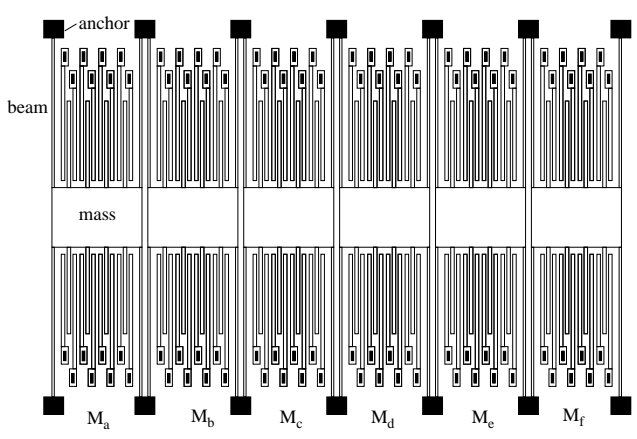

Fig. 4. Modularized comb accelerometer structure

module are connected to the modulation signal circuit through switches made of analog MUXes. By turning on or off these switches, we can determine whether a module works as part of the main device or the redundant device. For example, if the modulation signals of module $M_{c}$ are turned off, then the movable fingers of $M_{c}$ cannot sense any signal. Thus, $M_{c}$ is electronically disconnected from the MEMS device (though it is still connected to the entire MEMS structure physically), and is not involved in the MEMS function.

During the BISR mode, the device will first perform the BIST process for each individual module. The dual-mode BIST technology presented in [11] will be used here to ensure a thorough test. The BIST result of each module is compared with the pre-stored good module response to judge whether the module is good or faulty. This information is fed to the BISR control circuit as input for self repairing. If a module is tested as faulty, the control circuit will permanently exclude the module from the main device and replace it with a good redundant module (if there is any). Thus, after repairing, the main device can still be ensured to work properly.

In the following discussion, we call the MEMS comb accelerometer with (without) the BISR feature as a BISR (non-BISR) accelerometer. In order for a fair comparison, we assume that the total number of finger groups of the nonBISR accelerometer should equal to that of the main device in the BISR accelerometer. In this way, the area overhead of the modularized BISR accelerometer includes the area for two 
redundant modules, plus the area for extra beams and gaps between modules in the BISR accelerometer, as well as the area for control circuits.

\section{Built-in SElF-test of the ACCElerometer}

In order to implement the built-in self-repair of the accelerometer, each module need to perform a built-in self-test (BIST) on itself. The BIST results will be fed to the control circuit. The control circuit will construct main device from available good modules. If any module in the main device is tested to be faulty during in-field usage, the BISR control circuit will separate it out from the main device and replace it with a good redundancy. The BIST acts as the prerequisite for the BISR technique. A dual-mode BIST method [11] will be used here for the self-repairable accelerometer. The dualmode BIST technique partition the fixed instead of the movable capacitance plates, so that both sensitivity and symmetry BIST can be implemented on each module of the MEMS comb accelerometer. Since each of the sensitivity and symmetry BIST has its own fault coverage, a combination of both modes ensures a better fault coverage. The capacitance partition of a BISR module for dual-mode BIST implementation is shown in Figure 5. In order for simplification, only eight finger groups are shown in the module. In the figure, $M 1-M 8$ are movable fingers, $M s$ is the sensing mass, $D 1-D 8$ are driving fingers, and $S 1-S 8$ are sensing fingers.

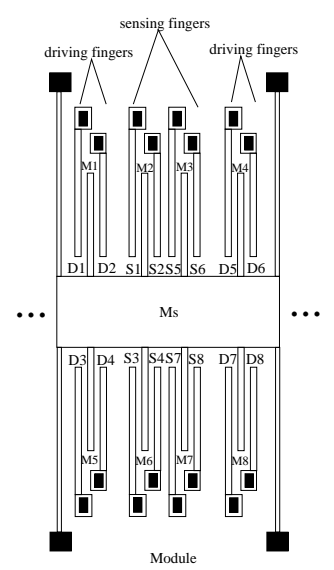

Fig. 5. Dual-mode BIST of one BISR module.

During normal operation, $\mathrm{TE}=0$, modulation voltage $V_{m p}$ is applied to $\{\mathrm{S} 1, \mathrm{~S} 3, \mathrm{~S} 5, \mathrm{~S} 7, \mathrm{D} 1, \mathrm{D} 3, \mathrm{D} 5, \mathrm{D} 7\}$, and $V_{m n}$ is applied to \{S2, S4, S6, S8, D2, D4, D6, D8 \}. The voltage level in the movable fingers $V_{M s}$ is measured as the output voltage to determine the acceleration. When $\mathrm{TE}=1$ and $\mathrm{TS}=0$, the device works in the sensitivity test mode. A certain test driving voltage $V_{d}$ is applied to $\{\mathrm{D} 1, \mathrm{D} 3, \mathrm{D} 5, \mathrm{D} 7\}$ to activate the device with electrostatic force. The modulation voltage $V_{m p}$ is applied to $\{\mathrm{S} 1, \mathrm{~S} 3, \mathrm{~S} 5, \mathrm{~S} 7\}$, while $V_{m n}$ is applied to $\{\mathrm{S} 2, \mathrm{~S} 4$, $\mathrm{S} 6, \mathrm{~S} 8\}$. The output voltage on movable mass Ms is measured for the device sensitivity. This value is compared with the expected good device value within a certain tolerance level to find whether the device is faulty. When $\mathrm{TE}=1$ and $\mathrm{TS}=1$, the device is in the symmetry test mode. Test driving voltage $V_{d}$ is applied to $\{\mathrm{D} 1, \mathrm{D} 3, \mathrm{D} 5, \mathrm{D} 7\}$, modulation voltage $V_{m p}$ is applied to $\{\mathrm{S} 1, \mathrm{~S} 5\}$, while $V_{m n}$ is applied to $\{\mathrm{S} 3, \mathrm{~S} 7\}$. The sensing circuit checks whether the output voltage on movable fingers is a constant zero to detect any asymmetry caused by local defects. If there is a non-zero voltage on the movable electrode Ms, then it indicates there are local defects which alter the symmetry of the device. The voltage biasing scheme for the comb accelerometer in the normal and both BIST modes is shown in Table I. Since each of the sensitivity BIST and symmetry BIST has its own fault coverage, a combination of both mode ensures better fault coverage.

TABLE I

VOLTAGE BIASING SCHEME FOR COMB ACCELEROMETER.

\begin{tabular}{|l|l|l|l|}
\hline \hline $\begin{array}{l}\text { Voltage } \\
\text { biasing }\end{array}$ & $\begin{array}{l}\text { Normal } \\
\text { operation }\end{array}$ & $\begin{array}{l}\text { Sensitivity } \\
\text { BIST }\end{array}$ & $\begin{array}{l}\text { Symmetry } \\
\text { BIST }\end{array}$ \\
\hline \hline$V_{d}$ & - & D1,D3,D5,D7 & D1,D3,D5,D7 \\
\hline$V_{n o m}$ & - & $\begin{array}{l}\text { D2,D4,D6,D8 } \\
\text { M1,M4,M5,M8 }\end{array}$ & $\begin{array}{l}\text { D2,D4,D6,D8 } \\
\text { M1,M4,M5,M8 } \\
\text { S2,S4,S6,S8 }\end{array}$ \\
& & & S1,S5 \\
\hline$V_{m p}$ & $\begin{array}{l}\text { S1,S3,S5,S7 } \\
\text { D1,D3,D5,D7 }\end{array}$ & S1,S3,S5,S7 & \\
\hline$V_{m n}$ & S2,S4,S6,S8 & S2,S4,S6,S8 & S3,S7 \\
& D2,D4,D6,D8 & & \\
\hline
\end{tabular}

To implement the BIST technique, a control circuit is needed to switch the device among the normal operation mode and both BIST modes. Such a control circuit is not complex and only contains some switches made of analog Muxes. The control circuit design for the BISR MEMS accelerometer is shown in Figure 6. In the control circuit, totally only six Muxes are needed: three 3-to-1 Muxes and three 2-to-1 Muxes. The differential capacitance detection circuit for BIST modes can be shared with that of the normal operation mode. Thus, the circuit overhead for the BIST technique implementation is small.

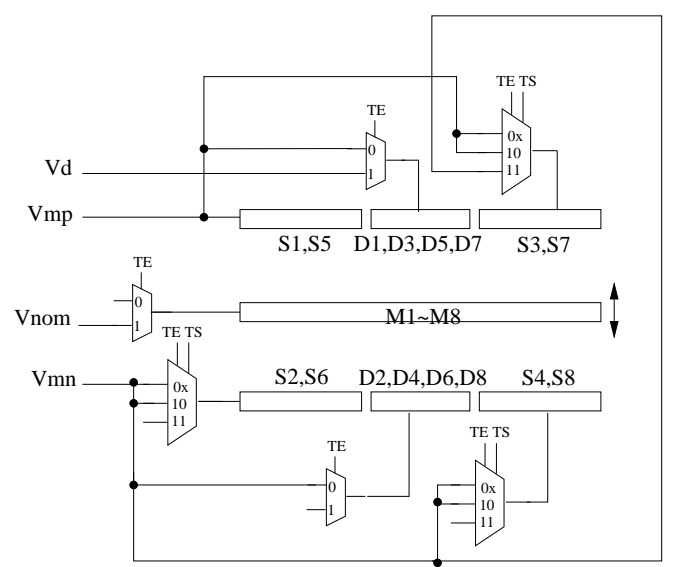

Fig. 6. The control circuit for dual mode BIST of one BISR module. 


\section{Performance Analysis}

\section{A. Sensitivity Analysis}

A MEMS comb accelerometer actually can be simplified by a spring-mass model. The width and length of each tether (seismic mass) are represented by $W_{b}\left(W_{m}\right)$ and $L_{b}\left(L_{m}\right)$ separately, while the width and length of each movable finger are denoted by $W_{f}$ and $L_{f}$ respectively. There are totally $N_{f}$ number of movable fingers, and the thickness of the device is $h$. Assume the density and the Young's modulus of poly-Si as $\rho$ and $E$ respectively. The sensing mass $M_{s}$ of the accelerometer, which includes the seismic mass and all the movable fingers attached to it, can be expressed as follows:

$$
M_{s}=\rho h\left(W_{m} L_{m}+N_{f} W_{f} L_{f}\right)
$$

The total spring constant $k_{t o t}$ of all four beams is:

$$
k_{t o t}=4 \cdot k_{s}=\frac{4 E h W_{b}^{3}}{L_{b}^{3}}
$$

The displacement sensitivity $S_{d}$ of the device, which is defined as the displacement of movable fingers per unit gravity acceleration ( $\mathrm{g}$ ) along the sensitive direction, can be expressed as:

$$
S_{d}=\frac{M_{s} g}{k_{t o t}}=\frac{\rho g\left(W_{m} L_{m}+N_{f} W_{f} L_{f}\right) L_{b}^{3}}{4 E W_{b}^{3}}
$$

Assume all other parameters are fixed, the relationships between the device displacement sensitivity $S_{d}$ and the beam width $W_{b}$, central mass width $W_{m}$ are shown in Figure 7.

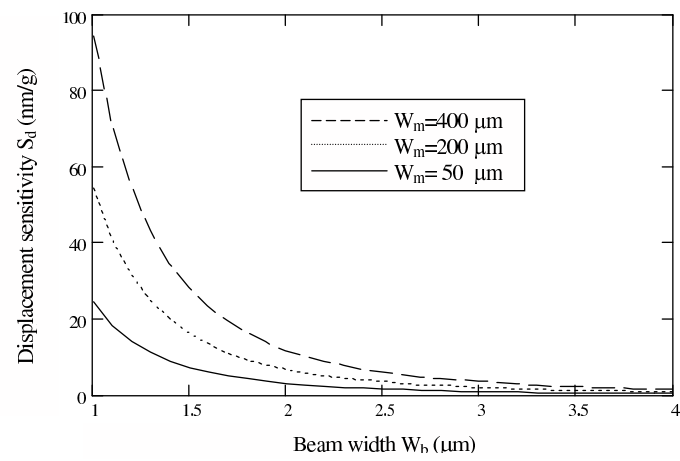

Fig. 7. The relationship between displacement sensitivity of accelerometer and beam/mass width

We denote the displacement sensitivity of the BISR (nonBISR) accelerometer by $S_{d b}\left(S_{d n b}\right)$. In the BISR device, we partition all $N_{f}$ comb finger groups into $n$ modules in the main device, and add $m$ number of modules as redundancy. That is, each BISR module contains $N_{f} / n$ comb finger groups. Correspondingly, the sensing mass of each BISR module also falls to $1 / n$ of the non-BISR version. If we keep the same geometry parameters (i.e., width $W_{b}$, length $L_{b}$ and thickness $h$ ) for the beams of both BISR and non-BISR designs, we have:

$$
S_{d b} \approx \frac{\frac{M_{s}}{n} g}{k_{t o t}}=\frac{1}{n} S_{d n b}
$$

where $M_{s}$ and $k_{t o t}$ are the sense mass and spring constant of the non-BISR accelerometer.
For the BISR accelerometer design, the sensitivity loss due to the modularized design can be recovered back by various methods, such as reducing the beam width of each module, using folded beams instead of straight beams, or enlarging the width $W_{m}$ of the central mass, etc. Among them, shrinking the beam width $W_{b}$ is the most efficient way for sensitivity compensation without changing the device area. Assume the beam width of the non-BISR accelerometer as $W_{b 0}$, for the case of $n=4$, if the beam width of the BISR accelerometer is reduced to $0.63 W_{b 0}$, the sensitivity of the BISR accelerometer will be fully compensated back to the nonBISR value. The designer can also compensate the sensitivity loss of the BISR accelerometer by the combination of the above methods according to the specific design requirements.

Even if all the device parameters (such as beam width and mass width, etc) are fixed, the sensitivity of an accelerometer in the open-loop mode can still be conveniently enhanced by electrostatic force with an appropriate DC biasing voltage [12]. The electrostatic force acts as a spring with a negative spring constant. This will help reduce the effective spring constant of the accelerometer and increase its sensitivity. Since this electrostatic force actually deflects the mass, it is equivalent to the effect of an inertial force acting on the mass. Thus, the electrostatic force is a powerful tool to enhance the sensitivity. A small DC biasing voltage (several volts) can increase the sensitivity to infinity, and this offers a great flexibility in the sensitivity recovery. Thus, if electrostatic force is used for sensitivity compensation, the beam width and mass width can be kept the same as those of the non-BISR device.

\section{B. Frequency Analysis}

Using the simplified spring-mass model discussed above for the comb accelerometer, we have the resonant frequency $f_{n s r}$ of the non-BISR design given by the following equation [7]:

$$
f_{n s r}=\frac{1}{2 \pi} \sqrt{\frac{k_{t o t}}{M_{s}}}
$$

where $M_{s}$ and $k_{t o t}$ are defined in the last section.

Assume the dimension of each beam in the BISR design remains the same as that of the non-BISR design, but the comb finger groups are divided into $n$ identical modules. The resonant frequency $f_{s r}$ of the BISR accelerometer can thus be given by

$$
f_{s r}=\frac{1}{2 \pi} \sqrt{\frac{k_{t o t}}{\frac{1}{n} M_{s}}}=\sqrt{n} f_{n s r}
$$

Further, the relationship between resonant frequency and displacement sensitivity can be given by:

$$
f_{n s r}=\frac{\sqrt{g}}{2 \pi \sqrt{S_{d}}}
$$

Consequently, if the displacement sensitivity of the BISR accelerometer is compensated to the same as that of the nonBISR design (e.g., by adjusting the $k_{t o t}$ value), the resonant frequency will also remain the same as that of the non-BISR device. 


\section{YIELD ANALYSIS OF BISR COMB ACCELEROMETER}

\section{A. Yield Model for MEMS Redundancy Repair}

Yield model for VLSI redundancy repair has been well developed [13][14]. However, yield model for MEMS redundancy repair has not been available. MEMS has its unique properties which are different from VLSI. For example, MEMS generally contains movable parts. These movable parts are vulnerable to various defect sources, such as stiction. However, such failure mechanism is not available in VLSI. Thus a yield model for MEMS redundancy repair must be developed. Assume a set of defects can occur to $N$ number of locations in a MEMS device, i.e., there are $N$ possible defects in the MEMS device. Further, assume every defect occurs independently of each other, and the probability for each defect to occur is equal and defined as $q$. Thus, based on the defect distribution discussed in [13], the probability $P(X=x)$ that $x$ number of indistinguishable randomly distributed defects occurring to the MEMS device can be expressed as a Poisson distribution:

$$
P(X=x)=\frac{\lambda^{x}}{x !} e^{-\lambda}
$$

The simple Poisson distribution is too pessimistic for yield estimation because the defect clustering effect is not considered. Hence, the compound Poisson distribution is more popular by considering the normalized distribution of a chip defect density clustering factor. The next problem is how the average defect $\lambda$ distributes. In this work, we assume that the defect distribution function $F(\lambda)$ for $\lambda$ is a gamma function given by the following equation [13]:

$$
F(\lambda)=\frac{1}{\Gamma(k)(A b)^{k}}(\lambda)^{k-1} e^{-\lambda /(A b)}
$$

where $A$ is the device area, $b$ is a defect density coefficient, $\lambda$ is the average defect, and $k$ is the clustering parameter. Further, the average defect density $D_{0}$ is given by:

$$
D_{0}=b \cdot k
$$

The probability that $x$ defects occur in a MEMS device with area $A$ can be given by the following equation [13]:

$$
\begin{gathered}
P(x, A)=\int_{0}^{\infty} \frac{\lambda^{x}}{x !} e^{-\lambda} F(\lambda) d \lambda \\
=\frac{1}{x ! \Gamma(k)(A b)^{k}} \cdot \int_{0}^{\infty}(\lambda)^{x+k-1} e^{-(1+1 / A b) \lambda} d \lambda \\
=\frac{\Gamma(x+k)(A b)^{x}}{x ! \Gamma(k)(1+A b)^{x+k}} \\
=\left(\begin{array}{l}
x+k-1 \\
x
\end{array}\right)\left(\frac{1}{1+A b}\right)^{k}\left(\frac{A b}{1+A b}\right)^{x}
\end{gathered}
$$

For a non-BISR MEMS accelerometer, the yield $Y_{0}$ is the probability that no defect occurs (i.e., $X=0$ ), which can be expressed as:

$$
Y_{0}=P\left(0, A_{0}\right)=\left(\frac{1}{1+A_{0} \cdot b}\right)^{k}
$$

where $A_{0}$ is the area of the non-BISR accelerometer. From this equation, we see that the yield drops as the device area

increase. This is reasonable because the larger the device area is, the more likely it may suffer from some defects.

Assume there are totally $i$ possible defects in the BISR MEMS accelerometer, $n$ number of modules in the main device, and $m$ number of redundant modules as shown in Figure 8. The yield of the BISR MEMS accelerometer after redundancy repairing, denoted as $Y_{r}$, equals the probability that none of the $i$ defects occurs, plus the probability that some among the $i$ defects do occur but they all fall into no more than $m$ number of modules. The former is the case where all the $(n+m)$ number of modules are healthy, while the latter is the case in which some modules are faulty, but the device can still be self-repaired into a good one through redundancy. Each of both cases will be investigated in the following discussions.

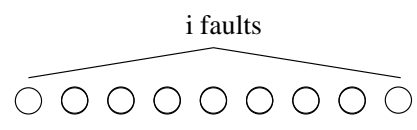

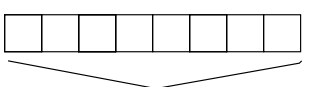

$\mathrm{n}$ main modules

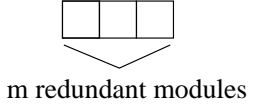

Fig. 8. Fault distribution among the modules of BISR accelerometer

Assume the main device of the BISR accelerometer has the same number of finger groups as the corresponding nonBISR design. Let $A_{0}$ denotes the area of the non-BISR accelerometer, $A_{b}$ denotes the area of beams in each module of the BISR accelerometer (and also the non-BISR accelerometer) plus the minimum gap between two modules/devices set by the design rules. In our simulation, we select $A_{b}=0.12 A_{0}$. The area $A_{r}$ of the BISR accelerometer is given by:

$$
\begin{aligned}
A_{r}= & \frac{(n+m)\left(A_{0}-A_{b}\right)}{n}+(n+m) A_{b} \\
= & \frac{(n+m)\left[A_{0}+(n-1) A_{b}\right]}{n}
\end{aligned}
$$

The probability that none of the $i$ number of defects occurs can be expressed as:

$$
P\left(0, A_{r}\right)=\left(\frac{1}{1+A_{r} \cdot b}\right)^{k}
$$

For the second case in which some defects do occur but the device can still be self-repaired through redundancy, it can be further divided into the following two sub-cases:

- The total number $(i)$ of defects is smaller than or equal to the number $(m)$ of redundant modules, i. e., $i \leq m$. For this sub-case, regardless of whatever distribution for the defects, the BISR comb accelerometer can always be repaired into a good device.

- The total number $(i)$ of defects is larger than the number of the redundant modules $m$; however, all the defects fall into $m$ number of modules or less. For this sub-case, the BISR accelerometer can still be repaired into a good device.

Both the above two sub-cases must be counted into the yield of the BISR accelerometer. The first sub-case can be 
easily solved, while the second sub-case requires an extensive analysis. For the first sub-case, the probability $P_{1}$ that $i$ number $(i \leq m)$ of defects occur in the BISR MEMS accelerometer can be expressed as:

$$
P_{1}=\sum_{x=1}^{x=m} P\left(x, A_{r}\right)
$$

For the second sub-case $(i>m)$, the faulty device can be repaired into a good device only if all the $i$ number of defects fall into $m$ number of modules or less. First, we examine the probability that $i$ defects are distributed into $j(j \leq m)$ MEMS modules and each of the $j$ modules contains at least one defect. So, we distribute one defect to each of the $j$ modules to ensure that each of the $j$ modules contains at least one defect. Thus, there are $i-j$ defects remaining to be distributed to the $j$ modules with any number of defects (maybe 0 ) for this distribution. As we know, there are $\left(\begin{array}{l}n_{0}+r-1 \\ r\end{array}\right)$ ways to distribute $r$ identical balls into $n_{0}$ distinct cells with any number of balls per cell. Here, we have $r=i-j$ and $n_{0}=j$ and the total number of ways of distribution is $\left(\begin{array}{c}i-1 \\ i-j\end{array}\right)$. Finally, the probability that the BISR accelerometer (with $n$ modules in the main device, and $m$ modules in the redundancy device) can be repaired when a certain $i$ number $(i>m$, sub-case 2$)$ of defects occur is:

$$
R(m, n, i)=\frac{\sum_{j=1}^{m}\left(\begin{array}{c}
m+n \\
j
\end{array}\right) \cdot\left(\begin{array}{l}
i-1 \\
i-j
\end{array}\right)}{\sum_{j=1}^{\min (i, m+n)}\left(\begin{array}{c}
m+n \\
j
\end{array}\right) \cdot\left(\begin{array}{l}
i-1 \\
i-j
\end{array}\right)}
$$

Thus, the probability $P_{2}$ that more than $m$ number of defects occur to a MEMS device, but it still can be self-repaired into a good device can be expressed as follows.

$$
\begin{aligned}
& P_{2}=\sum_{x=m+1}^{\infty} P\left(x, A_{r}\right) \cdot R(m, n, x) \\
& =\sum_{x=m+1}^{\infty} P\left(x, A_{r}\right) \cdot \frac{\sum_{j=1}^{m}\left(\begin{array}{l}
m+n \\
j
\end{array}\right) \cdot\left(\begin{array}{l}
x-1 \\
x-j
\end{array}\right)}{\sum_{j=1}^{\min (x, m+n)}\left(\begin{array}{l}
m+n \\
j
\end{array}\right) \cdot\left(\begin{array}{l}
x-1 \\
x-j
\end{array}\right)}
\end{aligned}
$$

Hence, the yield for the BISR accelerometer after redundancy repair is:

$$
\begin{aligned}
& Y_{r}(m, n)=P\left(0, A_{r}\right)+P_{1}+P_{2}
\end{aligned}
$$

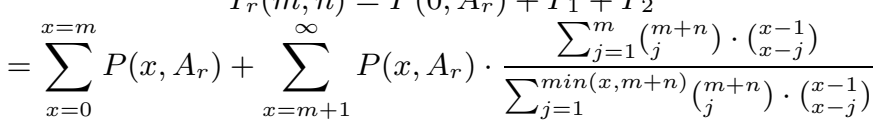

The yield increase $I Y(m, n)$ of the BISR accelerometer, when compared with the corresponding non-BISR accelerometer, can be given by:

$$
\begin{gathered}
I Y(m, n)=Y_{r}(m, n)-Y_{0} \\
=\sum_{x=0}^{x=m} P\left(x, A_{r}\right)+\sum_{x=m+1}^{\infty} P\left(x, A_{r}\right) \cdot \frac{\sum_{j=1}^{m}\left(\begin{array}{l}
m+n \\
j
\end{array}\right) \cdot\left(\begin{array}{l}
x-1 \\
x-j
\end{array}\right)}{\sum_{j=1}^{\min (x, m+n)}\left(\begin{array}{c}
m+n \\
j
\end{array}\right) \cdot\left(\begin{array}{l}
x-1 \\
x-j
\end{array}\right)} \\
-P\left(0, A_{0}\right)
\end{gathered}
$$

We can see that under this theory, if we set $m=0$ and $n=1$, then $Y_{r}(0,1)$ comes back to $P\left(0, A_{0}\right)$ which is exactly the yield of the non-BISR device. Thus, the general case of $Y_{r}(m, n)$ includes the yield of the non-BISR device as a special case.

Based upon the MEMS yield model for redundancy repair, we can derive the relationship between the yield increase and the non-BISR device yield for different $m$ and $n$ numbers. Figure 9 shows the simulation result for $n=4$ and $m=$ $2,4,6,8$ separately. From this figure, we can observe that the BISR device always gives a positive yield increase regardless of the non-BISR yield. This demonstrates the effectiveness of the redundancy repair technique for MEMS devices. If the non-BISR yield is too low (approaches 0) or too high (approaches 1), the yield increase by redundancy repair is not significant. This is a reasonable result. For a very low nonBISR yield (approaches 0), the defect density is extremely high and there are too many faulty modules in the main device. Compared with so many defective modules in the main device, the redundancy is relatively deficient to repair all of them. Thus, the yield increase by redundancy BISR is not significant. For a very high non-BISR yield (approaches 1), the main device itself is highly likely to be fault-free, and hence repair is not necessary.

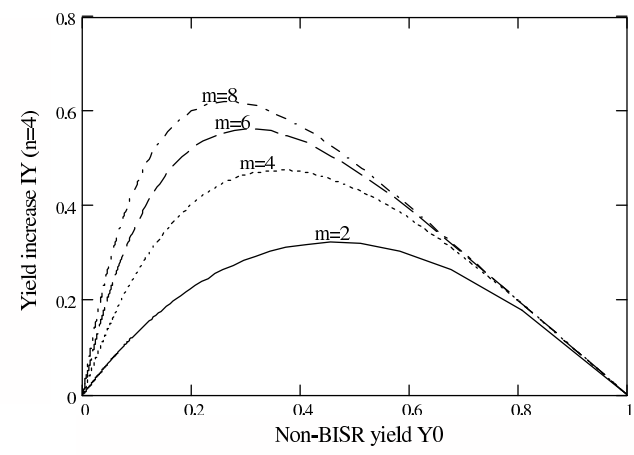

Fig. 9. The yield increase vs. non-BISR yield for different $\mathbf{m}$ numbers

In order to verify the effectiveness of redundancy repair for moderate initial yield, we randomly select $A_{0}=0.24 \mathrm{~mm}^{2}$, $b=1.8 / \mathrm{mm}^{2}$, and $k=1$, the yield of the non-BISR accelerometer is 0.698. For the BISR accelerometer with $m=$ 2 and $n=4$, the yield becomes 0.947 which is an increase of $35.7 \%$ (when compared with the non-BISR yield 0.698). This demonstrates an effective improvement on the yield for a comb accelerometer through redundancy repair.

\section{DESIGN AND SimUlation OF BISR ACCELEROMETER}

The geometry parameters of the BISR comb accelerometer with $m=2$ and $n=4$ are listed in Table II, using a set of design rules comparable to ADXL accelerometers [7]. For comparison, a none-BISR accelerometer with the same number of capacitance groups (as that at the main device of the BISR accelerometer) is also designed. The geometry parameters of the non-BISR accelerometer are also listed in the same table. The simulation results for the performance of both BISR and none-BISR accelerometers are shown in Table III. From Table III, we can see that, by narrowing the beam width from $3 \mu m$ (non-BISR beam width) to $2 \mu m$ (BISR beam width), the sensitivity loss of the BISR accelerometer due to device modularization can be fully compensated. The BISR device 
demonstrates a displacement sensitivity of $9.3 \mathrm{~nm} / \mathrm{g}$, which is even larger than that of the non-BISR device $(8.1 \mathrm{~nm} / \mathrm{g})$. This shows that the sensitivity loss can be effectively compensated by shrinking the beam width of the comb accelerometer.

TABLE II

Design of BISR/non-BISR accelerometers

\begin{tabular}{|l|l|l|}
\hline \hline $\begin{array}{l}\text { Design } \\
\text { Parameters }\end{array}$ & $\begin{array}{l}\text { BISR } \\
\text { device }\end{array}$ & $\begin{array}{l}\text { non-BISR } \\
\text { device }\end{array}$ \\
\hline \hline device area $\left(\mu m^{2}\right)$ & $1500 \times 900$ & $980 \times 900$ \\
\hline thickness t $(\mu m)$ & 6 & 6 \\
\hline no. of capacitance groups & $20 \times 6$ & 80 \\
\hline capacitance gap $d_{0}(\mu m)$ & 2 & 2 \\
\hline beam width $W_{b}(\mu m)$ & 2 & 3 \\
\hline beam length $L_{b}(\mu m)$ & 300 & 300 \\
\hline mass width $W_{m}(\mu m)$ & 300 & 200 \\
\hline mass length $L_{m}(\mu m)$ & $220 \times 6$ & 880 \\
\hline fi nger width $W_{f}(\mu m)$ & 4 & 4 \\
\hline fi nger length $L_{f}(\mu m)$ & 200 & 200 \\
\hline
\end{tabular}

TABLE III

Simulation results of BISR/non-BISR accelerometers

\begin{tabular}{|l|l|l|}
\hline \hline Performance & $\begin{array}{l}\text { BISR } \\
\text { main device }\end{array}$ & $\begin{array}{l}\text { non-BISR } \\
\text { device }\end{array}$ \\
\hline \hline sensing mass $M_{s}(\mu \mathrm{g})$ & $1.15 \times 4=4.6$ & 3.4 \\
\hline capacitance $C_{0}(\mathrm{pF})$ & $0.1 \times 4=0.4$ & 0.4 \\
\hline sensitivity $S_{d}(\mathrm{~nm} / \mathrm{g})$ & 9.3 & 8.1 \\
\hline sensitivity $S_{c}(\mathrm{fF} / \mathrm{g})$ & $0.96 \times 4=3.84$ & 3.3 \\
\hline spring constant $k_{m}(\mathrm{~N} / \mathrm{m})$ & $1.21 \times 4=4.84$ & 4.08 \\
\hline frequency $f_{0}(\mathrm{kHz})$ & 5.17 & 5.55 \\
\hline
\end{tabular}

\section{CONCLUSIONS}

In this paper, a self-repairable MEMS comb accelerometer device design is proposed. The strategy of the BISR device is to partition the large number of comb fingers into several modules. Thus each module contains less number of comb fingers, the risk of being faulty is reduced. Further, redundant modules are introduced. If any of the working module is found to be faulty during the BIST, it will be replaced with a good redundant module. In this way, the device can be self-repaired into good one. The modularized design leads to a sensitivity loss. However, it can be compensated by adjusting the design parameters, such as shrinking the beam width, enlarge the mass width, etc. Even if all the design parameters are already fixed, a powerful electrostatic force compensation method can still be used to fully recover the device sensitivity without changing the device parameters. The built-in self-test of each BISR module is discussed. In order to quantitatively evaluate the effectiveness of the BISR technique on the yield increase, a yield model for MEMS redundancy repair is developed. The simulation results based on the yield model demonstrate an effective yield increase due to the redundancy repair. The yield increase is especially effective for moderate initial yield. However, if the initial yield is too large or too small, the yield increase due to redundancy repair is not significant. For demonstration purpose, for a moderate initial yield of 0.626 , the yield after repair is $92.5 \%$. A yield increase of 33.5\% can be achieved due to redundancy repair. Compared to the "hard" repair with physical or chemical process, the redundancy repair has the advantage of low cost, suitable for in-field usage, etc. Furthermore, it is virtually applicable to any local defect within one module. This is because if any of the module is faulty due to local defect, it will be separated out from the main device and replaced with a good redundant module. However, the proposed BISR technique cannot be applied to global defects. For global defects, all the modules are faulty and no good module are available. Thus, there will be no good redundant module available for repair.

Due to the fabrication variation, the performance of the MEMS accelerometer modules cannot be perfectly the same. During the BISR, when the faulty module is replaced with good redundancy, the tiny performance difference between the switched modules must be compensated. Our future research is to develop a built-in self-calibration (BISC) technique to ensure a smooth switch between the defective and healthy modules, so that the device performance will maintain the same after redundancy repair.

\section{ACKNOWLEDGEMENT}

The authors are thankful to Dr. Tarek Sobh, Dr. Lawrence Hmurcik and Dr. Stephen Grodzinsky in University of Bridgeport for their valuable discussions in the paper.

\section{REFERENCES}

[1] G. T. A. Kovacs, Micromachined Transducers Sourcebook, WCB/McGraw-Hill, New York, 1998.

[2] URL: http://public.itrs.net.

[3] K. Chakraborty and P. Mazumder, Fault-Tolerance and Reliability Techniques for High-Density RAMs, Prentice Hall, Upper Saddle River, NJ 07458, 2002.

[4] B. Johnson, Design and Analysis of Fault Tolerant Digital Systems, Addison Wesley, 1989.

[5] J. Ramirez-Angulo, and R. L. Geiger, 'New Laser-trimmed Film Resistor Structures for Very High Stability Requirements", IEEE Transactions on Electron Devices, Vol. 35, Issue 4, pp. 516-518, Apr. 1988.

[6] K. Tanaka, Y. Mochida, M. Sugimoto, K. Moriya, T. Hasegawa, K. Atsuchi, and K. Ohwada, "A Micromachined Vibrating Gyroscope", Sensors and Actuators A: Physical, Vol. 50, pp. 111-115, 1995.

[7] W. Kuehnel, and S. Sherman, "A Surface Micromachined Silicon Accelerometer with On-chip Detection Circuitry," Sensors and Actuators A: Physical, Vol. 45, pp. 7-16, 1994.

[8] "ADXL150/ADXL250: $\pm 5 \mathrm{~g}$ to $\pm 50 \mathrm{~g}$, Low Noise, Low Power,Single/Dual Axis iMEMS Accelerometers", ADXL150/ADXL250 datasheet, URL: http://www.analog.com.

[9] X. Xiong, D. Lu, and W. Wang, "A Bulk-micromachined Comb Accelerometer with Floating Interconnects", Proceedings of 48th IEEE International Midwest Symposium on Circuits and Systems (MWSCAS'05), Cincinnati, Ohio, Aug 7-10, 2005, accepted to be published.

[10] N. Deb, and R. D. Blanton, "Analysis of Failure Sources in Surfacemicromachined MEMS", Proc. of International Test Conference, pp. 739-749, Oct. 2000.

[11] X. Xiong, Y. Wu, W. Jone, "A Dual-Mode Built-In Self-Test Technique for Capacitive MEMS Devices", Proc. of the 22nd IEEE VLSI Test Symposium (VTS'04), pp. 148-153, May 25-29, 2004.

[12] B. Li, D. Lu, and W. Wang, 'Open-loop Operating Mode of Micromachined Capacitive Accelerometer", Sensors and Actuators A: Physical, Vol. 79, pp. 219-223, 2000.

[13] J. Hirase, 'Yield Increase of VLSI after Redundancy-repairing," Proc. of 10th Asian Test Symposium, pp.353-358, Nov. 2001.

[14] T. L. Michalka, R. C. Varshney, and J. D. Meindl, "A Discussion of Yield Modeling with Defect Clustering, Circuit Repair, and Circuit Redundancy", IEEE Transactions on Semiconductor Manufacturing, Vol. 3, Issue 3, pp.116-127, Aug. 1990. 